\title{
Unraveling the Correlations between Conformation, Lubrication and Chemical Stability of Bottlebrush Polymers at Interfaces
}

Jimmy Faivre ${ }^{1,3}$, Buddha Ratna Shrestha ${ }^{1}$, Guojun Xie, ${ }^{2}$ Thierry Delair ${ }^{3}$, Laurent David $^{3}$, Krzysztof Matyjaszewski $^{2 *}$, Xavier Banquy $^{1 *}$

${ }^{1}$ Canada Research Chair in Bioinspired materials, Faculty of Pharmacy, Université de Montréal, Montréal, Qc, Canada

${ }^{2}$ Center for Macromolecular Engineering, Department of Chemistry, Carnegie Mellon University, Pittsburgh, PA, USA

${ }^{3}$ Université de Lyon, Université Claude Bernard Lyon 1, CNRS, Ingénierie des Matériaux Polymères (IMP-UMR 5223), 15 Boulevard Latarjet, 69622 Villeurbanne Cedex, France 


\begin{abstract}
In the present study, we monitored the conformation and chemical stability of a hydrophilic bottlebrush (BB) polymer in pure water and buffered saline solutions. We correlated these parameters to lubricating and wear protecting properties. Using the Surface Forces Apparatus (SFA), we show that the BB polymer partially adsorbs on mica surfaces and extends half its contour length toward the aqueous media. This conformation gives rise to a strong repulsive interaction force when surfaces bearing BB polymer chains are pressed against each other. Analysis of these repulsive forces demonstrated that the adsorbed polymer chains could be described as end-attached elastic rods. After 2 months of aging at temperatures ranging from 4 to $37{ }^{\circ} \mathrm{C}$, partial scission of the BB polymer's lateral chains was observed by Gel Permeation Chromatography with a half-life time of the polymer of at least two years. The thickness of the BB polymer layer assessed by SFA appeared to quickly decrease with aging time and temperature which was mainly caused by the adsorption to the substrate of the released lateral chains. The gradual loss of the BB polymer lateral chains did not impact significantly the tribological properties of the BB polymer solution nor its wear protection capacity. The friction coefficient between mica surfaces immersed in the BB polymer solution was $\mu=0.031 \pm 0.002$, and was independent of the aging conditions and remained constant up to an applied pressure $\mathrm{P}=$ 15 atm. Altogether, this study demonstrates that besides the gradual loss of lateral chains, the BB polymer is still able to perform adequately as a lubricant and wear protecting agent over a time period suitable for in vivo administration.
\end{abstract}

Keywords: Bottlebrush polymer, stability, wear protection, polymer conformation 


\section{Introduction}

Bottlebrush (BB) polymers are composed of a linear macromolecular backbone to which are densely grafted side chains ${ }^{1}$. The strong steric repulsion between side chains leads to the backbone extension resulting in a worm-like conformation of the polymer which can be modulated by side-chain grafting density and length ${ }^{1,2}$. BB polymers have attracted a growing interest in many application fields ranging from materials to biomedical engineering ${ }^{3}$. Indeed, grafted or adsorbed BB polymers exhibit exceptional antifouling ${ }^{4}$, viscoelastic ${ }^{5}$, lubrication ${ }^{6}$, surface coating ${ }^{7}$ and photonic ${ }^{8}$ properties. BB polymers combined with contrast agents have also demonstrated exceptional properties as diagnostic tools for MRI and fluorescence imaging 9 . Recent reports are now suggesting the use of BB polymers as drug delivery systems ${ }^{10}$. Contrarily to spherical polymeric particles which have been extensively studied, cylindrical polymer brushes have demonstrated longer blood circulation time ${ }^{11,12}$ and improved biodistribution ${ }^{13}$, revealing them as promising drug carriers. The BB architecture is abundantly present in nature, in particular in proteins such as mucins and proteoglycans found in articular joints or in the gastro intestinal tract. First reported in natural systems including the protein lubricin ${ }^{14-19}$ or agrecans $^{20-22}$ and later in synthetic systems ${ }^{6,23-27}$, the BB architecture is well known to provide excellent friction reduction capabilities to lubricating polymers.

Three main synthetic routes exist to prepare polymeric molecular brushes: the "grafting to" polymerization via the coupling reaction of an end-functionalized side-branch to a polymeric backbone; the "grafting through" polymerization of macromonomers bearing the pendant chains and the "grafting from" polymerization of monomers from a macroinitiator ${ }^{3}$. These techniques can be implemented to grow polymer chains on surfaces or in solution. This allows to obtain a 
rich variety of brushes besides the fact that these strategies are challenging to implement due to the steric hindrance between lateral chains and the stress it creates on the BB polymer backbone.

BB polymers chemical stability at surfaces or in solution have been studied extensively ${ }^{28-30}$. Different mechanisms leading to BB chemical degradation have been identified. Sheiko et al. reported carbon-carbon bond scissions at the backbone of hydrophobic BB polymers deposited on flat surfaces ${ }^{29}$. The authors attributed this phenomenon to an increased backbone tension originated by the spreading of the densely grafted side chains on the substrate. Xia et al. observed the same phenomenon with ultrahigh molecular weight cyclic BB polymer deposited on graphite ${ }^{30}$. Chen et al. reported that end-grafted polystyrene (PS) chains in the brush conformation can also suffer chain scission ${ }^{31}$. The authors explained their results invoking intermolecular transfer reactions which occurred at higher rates for PS brushes compared to free PS chains due to their close packing. Higher degradation rates were also reported for grafted poly(lactic acid) (PLA) brushes compared to bulk PLA ${ }^{32}$. In this case, the degradation mechanism was mediated by intramolecular transesterification and ester hydrolysis ${ }^{32}$. Naturally occurring BB macromolecules such as proteoglycans have been reported to undergo degradation as well via proteolysis. This is particularly the case in adult articular joints where the aggrecan, a densely grafted proteoglycan acting as a joint lubricant, suffers from fragmentation by enzymatic chain scission ${ }^{33}$. This phenomenon can be accelerated in an inflamed environment such as arthritic articular joints where specific enzymes degrade chondroitin and keratan sulfates glycosaminoglycans side chains.

So far, only a few studies have investigated the changes in BB polymers interfacial properties along with their time-dependent structural changes. In this manuscript, we intent to relate the interfacial properties (normal interaction forces and lubrication forces) of BB polymers to their 
chemical stability. We used a BB polymer known to exhibit excellent lubricating properties (friction coefficient $\mu \sim 0.02$ ) anti-wear properties (pressure at onset of damage $>3 \mathrm{MPa}$ ) when combined with high molecular weight hyaluronic acid $(\mathrm{HA})^{34}$. We first used the surface forces apparatus (SFA) to characterize the interfacial properties of the BB polymer. The wear protection capacity and the lubricating properties of BB polymer solutions as well as the interaction forces between mica surfaces across such solutions were monitored under different aging conditions and correlated to the chemical stability of the polymer. The chemical stability of the BB polymer was assessed by estimating the side chains grafting density using the SFA, the BB backbone contour length by atomic force microscopy (AFM) and the molecular weight of the BB polymer using GPC. 


\section{Materials and methods}

Polymer synthesis and Formulation. The synthesis of the zwitterionic BB polymer was performed as previously reported ${ }^{34}$. The polymer was synthesized by atom transfer radical polymerization $(\mathrm{ATRP})^{35,36}$, purified by dialysis in pure water prior to recovery. For all stability studies, the BB polymer (white powder) was dissolved at $100 \mu \mathrm{g} / \mathrm{mL}$ in phosphate buffered saline (PBS, $150 \mathrm{mM} \mathrm{NaCl}, \mathrm{pH}=7.4$ ) and left in solution in a dark container at $4{ }^{\circ} \mathrm{C}, 22^{\circ} \mathrm{C}$ or $37^{\circ} \mathrm{C}$.

AFM imaging. At different time points, aliquots of BB polymer solutions were diluted with pure water up to a concentration of 50 to $5 \mu \mathrm{g} / \mathrm{mL}$ and deposited on freshly cleaved mica. The BB polymer was left to adsorb for 20 min then exceeding solution was removed using a kimwipe and the surface was washed several times with water to completely remove remaining salt cristals. The surface was air dried prior to imaging. For imaging, a Multimode Dimension 3100 AFM equipped with nanoscope VIII controller (Digital instruments) was used in the peak force QNM mode. Scanasyst-air silicon tips were used for imaging. The contour length of the BB polymer was obtained by analysing AFM images using WormTracker plug-in for ImageJ ${ }^{37}$.

Interaction forces. A Surface Forces Apparatus (SFA 2000, SurForce LLC, USA) was used to measure the interaction force profiles between mica surfaces across BB solutions. Briefly, backsilvered mica sheets were glued (epoxy glue Epon 1004F) on glass cylindrical disks with a curvature, $R$, of $2 \mathrm{~cm}$ under a laminar flow hood. The disks were then mounted in the SFA chamber in a cross configuration. The SFA chamber was then purged with dry argon and the surfaces were brought into adhesive contact to quantify the mica surfaces' thickness. The surfaces were then separated again and $50 \mu \mathrm{L}$ of BB polymer solution were injected between the 
surfaces. To avoid evaporation of the solution during experiment, pure water was deposited at the bottom of the chamber to saturate the closed atmosphere. The setup was left to equilibrate for $1 \mathrm{~h}$ before measurements were started. The separation distance between the two opposing mica surfaces was determined by multiple beam interferometry. The wavelength of the Fringes of Equal Chromatic Order (FECO) was measured with a spectrometer and converted into separation distance using the three layer interferometer $\operatorname{model}^{38}$. The normal interaction forces, $F_{\mathrm{N}}$, were recorded as a function of separation distance, $D$, for in (compression) and out (separation) runs at a speed of $1 \mathrm{~nm} / \mathrm{s}$. The fringes were analyzed using a in-house Matlab® routine. Experiments were performed at least three times at different contact positions.

Tribological experiments. The friction forces, $F_{\mathrm{t}}$, as a function of normal force, $F_{\mathrm{N}}$, were measured using the friction device and bimorph slider ${ }^{39}$. As in the interaction forces section, 50 $\mu \mathrm{L}$ BB polymer solution were injected between the surfaces and a small amount of pure water was added in the chamber to limit evaporation. The setup was left to equilibrate for $1 \mathrm{~h}$ before experimentation. To ensure reproducible BB polymer surface coverage, normal force profiles measurements were carried out right before tribotesting. The normal force was monitored using semi-conductive strain gauges mounted on the double cantilever of the bimorph slider. Friction and normal forces were recorded on a digital recorder (Soltec TA220-2300A). The sliding velocity was fixed at $2.5 \mu \mathrm{m} / \mathrm{s}$ using a Function/Arbitrary waveform generator (Agilent 33250A). The amplitude of the sliding motion was set to $50 \mu \mathrm{m}$. The separation distance, $D$, wear initiation and surface contact area were simultaneously monitored during tribological experiments by continuously recording the FECO using a CCD camera ${ }^{40}$.

Gel Permeation Chromatography (GPC). Monitoring of the molecular weight and dispersity of the BB polymer was assessed by GPC with an eluting phase of $10 \mathrm{mM}$ phosphate aqueous 
buffer at $\mathrm{pH}$ 7.4. The GPC was equipped with a light scattering HELEOS (Wyatt), refractive index detector rEX, Wyatt QELS+ and UV detectors and a PL aquagel OH M 8 micro and H 8 micro columns. The flow rate was set at $0.5 \mathrm{~mL} / \mathrm{min}$ and the system was systematically equilibrated at $25^{\circ} \mathrm{C}$ for all measurements. The value of the $\mathrm{BB}$ polymer refractive index increment, $\mathrm{dn} / \mathrm{dc}$, was set to $0.142 \mathrm{~mL} / \mathrm{g}^{41}$. 


\section{Results and discussion}

The chemical composition of the BB polymer was $\left(\mathrm{PBiBEM}_{459^{-}}\right.$- $\left.-\mathrm{PMPC}_{35}\right)$-stat-PMMA 370 (see figure 1A). A similar polymer was recently shown to have excellent lubricating properties with a friction coefficient close to $10^{-2}$ under a normal pressure of 15 atm when used in combination with high molecular weight hyaluronic acid in buffered saline. ${ }^{34}$ These properties make this polymer a promising candidate for biomedical applications such as viscosuplementation of osteoarthritic joints to re-establish wear protection and possibly decelerate cartilage erosion. The architecture of the BB polymer used in this study, shown in figure $1 \mathrm{~A}$ and $\mathrm{B}$, mimics the lubricating proteins encountered in synovial joints ${ }^{6,42}$ such as lubricin and aggrecans. The contour length of the BB polymer, measured by AFM imaging, was found to be $141 \pm 25 \mathrm{~nm}$. Figure $1 \mathrm{C}$ represents a perpendicular cross-sectional profile of the BB polymer deposited on a

A

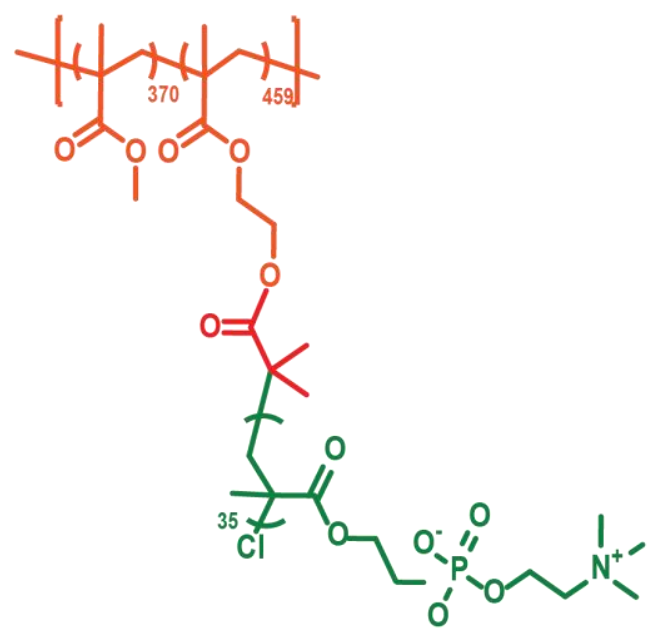

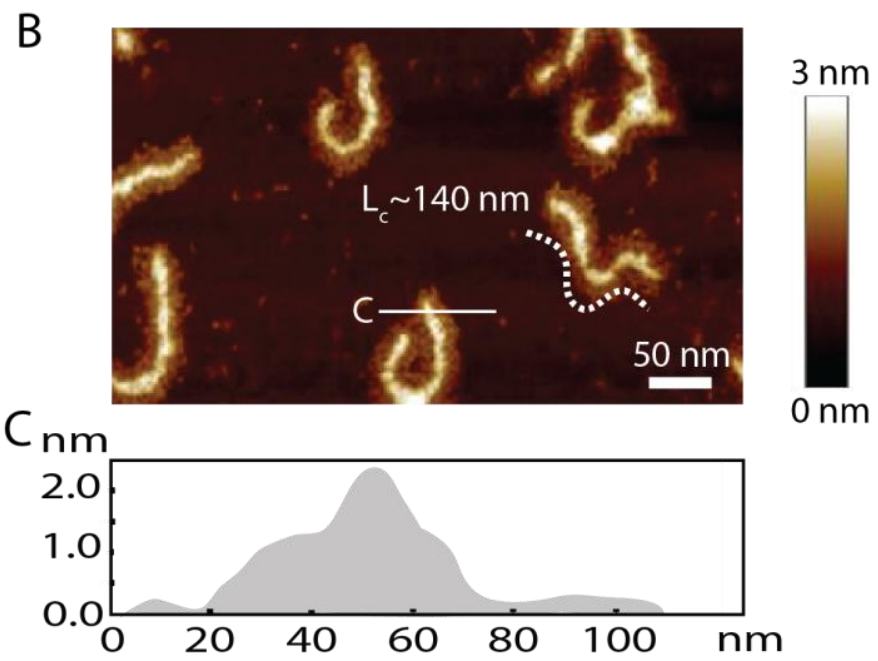

Figure 1. (A) Chemical structure of the bottle-brush polymer used in the study. (B) AFM picture of BB polymer deposited on mica surfaces from a $10 \mu \mathrm{g} / \mathrm{mL}$ polymer solution. (C) BB height profile of a perpendicular cross-section indicated with the white line in fig. 1B showing the contour of the polymer. mica substrate. The hydrophilic side-

side chains of the polymer appeared to spread on the surface suggesting a favorable affinity towards the surface. 


\section{Interaction forces across BB polymer solutions}

We used the SFA to measure the interaction profiles across BB polymer solutions in water or in saline solutions (Fig. 2A). As can be seen in Fig. 2B, repulsive forces were recorded during the in (surfaces approach) and out (surfaces separate) runs. At a fixed BB polymer concentration of $100 \mu \mathrm{g} / \mathrm{mL}$, the profiles were identical, independently of the ionic strength of the medium, as previously observed for polyzwitterionic brushes. ${ }^{34,43,44}$ The onset of the interaction forces, given by the separation distance at which adsorbed BB chains facing each other start interacting

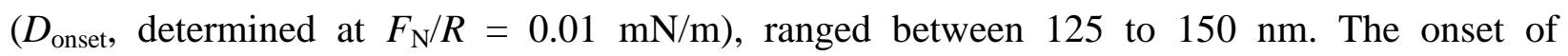
interaction being equal to twice the adsorbed polymer layer thickness, $L$ ( $L=62-75 \mathrm{~nm}$, no interdigitation hypothesis, see schematic Fig. 2C) and the average contour length of the polymer $L_{\mathrm{c}}$ being equal to $\sim 140 \pm 20 \mathrm{~nm}$ (assessed by AFM), we can conclude that adsorbed BB polymer chains extend on average half their contour length toward the medium. Under high pressure, the BB polymer film is about $5 \mathrm{~nm}$ thick, indicating the presence of at least one molecular layer of polymer.
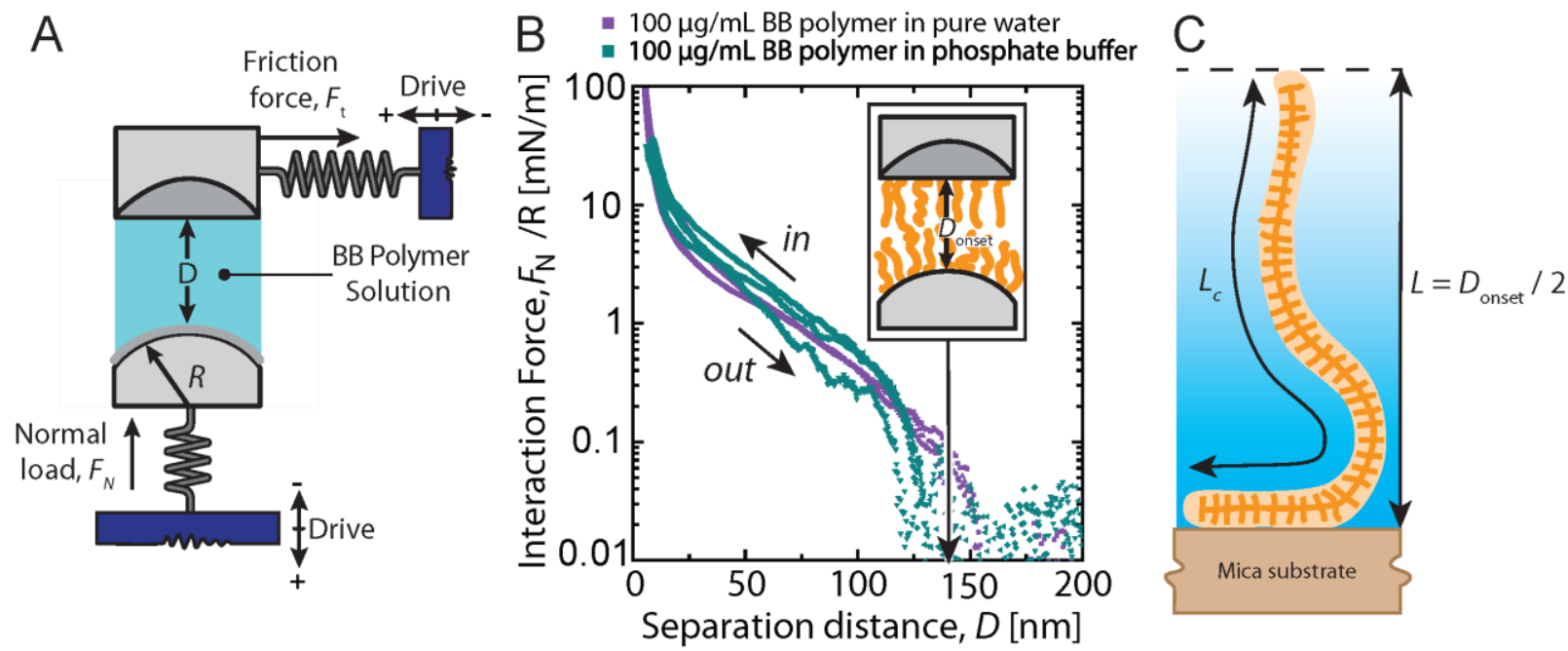

Figure 2. (A) SFA experimental setup used for normal force profiles and tribology experiments. (B) Interaction force profiles across BB solution at $100 \mu \mathrm{g} / \mathrm{mL}$ in pure water and in a phosphate buffer at 
$150 \mathrm{mM} \mathrm{NaCL}$ and $\mathrm{pH}$ 7.4. C) Schematic representation of the BB polymer conformation on the mica substrate with its characteristic dimensions.

In order to determine the conformation and interfacial properties of the polymer, we performed measurements of interaction forces between mica surfaces across solutions of BB polymer at two different concentrations (10 and $100 \mu \mathrm{g} / \mathrm{mL}$ ) and two different saline conditions (pure water and PBS). The measured interaction forces shown in Figure 3A present features common to all tested conditions. The force profiles $\left(F_{\mathrm{N}} / R\right.$ vs $\left.D\right)$ were once again purely repulsive even at $10 \mu \mathrm{g} / \mathrm{mL}$ where the surfaces were not fully covered (Figure 1B) which could have facilitated polymer bridging. Another interesting common feature is the constant onset of interaction forces for all polymer concentrations or saline conditions tested. Such behavior is quite unusual since the conformation, and therefore the polymer layer thickness, of randomly adsorbed or end-grafted linear polymer chains' conformation is known to depend on chain surface density. ${ }^{45}$ 

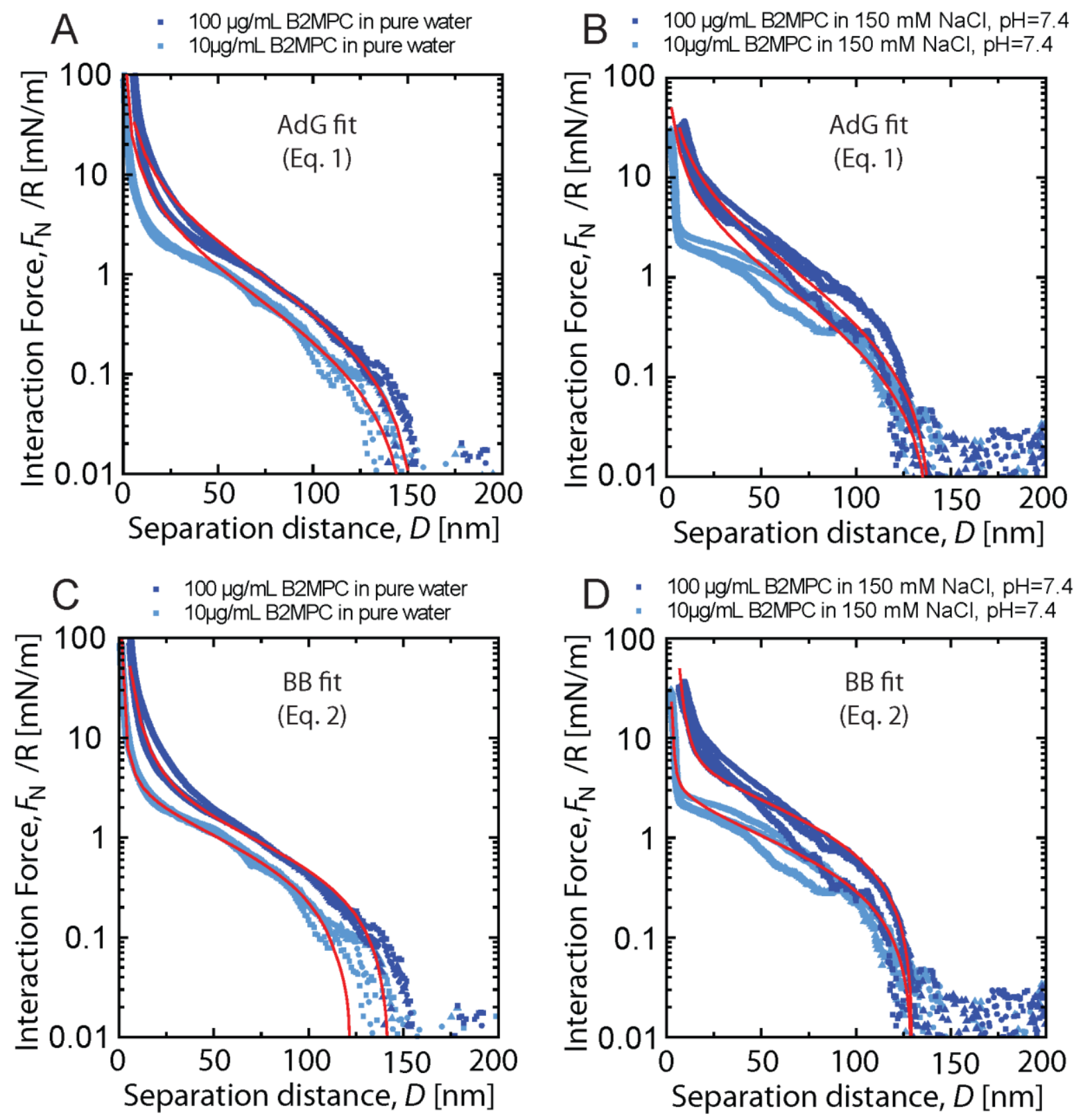

Figure 3. (A) Interaction force profile between mica surfaces across a solution of BB polymer in pure water $(A$ and $C)$ and in buffered saline ( $B$ and $D)$. The red lines are the best fitted curves using Eq. 1 (AdG fit) and Eq. 2 (in the manuscript, BB fit).

Reports on interaction forces between BB polymer coated surfaces are scarce, therefore analysis of the present experimental force profiles needs particular attention. In order to analyse the interaction profiles, we used two different theories: the Alexander-de Gennes (AdG) brush 
theory $^{46}$ (Eq. 1) and a theory based on the entropy and bending energies of BB polymers (BB model, Eq. 2).

In the AdG brush theory, the interaction energy between two flat surfaces bearing polymer brushes, $W$, which is related to the interaction force via the Derjaguin approximation $\left(F_{\mathrm{N}}=\right.$ $2 \pi \mathrm{R} W$ ) has two main contributions: an osmotic contribution that increases as separation distance between the surfaces decreases due to concentration of polymer segments, and an elastic contribution that decreases with the separation distance. The resulting force law reads:

$$
\frac{F_{N}}{R}=\frac{16 \pi k_{B} T L}{35 s^{3}}\left[7\left(\frac{2 L}{D}\right)^{5 / 4}+5\left(\frac{D}{2 L}\right)^{7 / 4}-12\right] \quad \text { (Eq. 1) }
$$

where $=1 / \sqrt{\pi \Gamma}$.

The AdG model has been shown to apply to many different systems, not only to end grafted brushes. Studies have demonstrated that the AdG model describes correctly the density profile and interaction profiles between end-adsorbed diblock polymers ${ }^{47}$. It also described fairly well the density profile of polymer loops and their interaction forces ${ }^{48}$. Interaction forces between surfaces bearing lubricin followed the AdG model as well. ${ }^{14}$ Therefore we can assume that the same model could apply to the present system. The tests performed at different polymer concentrations demonstrated that the AdG model was not able to fit satisfactorily the data in the whole range of concentration studied (Fig. 3A and B). Indeed, from the data provided in Table 1, we can immediately see that AdG model does not fit satisfactorily the experimental data at low polymer concentration. The force profiles also demonstrate that the onset of interaction is insensitive to the polymer solution concentration (and therefore to the surface density as well) which is inconsistent with the AdG brush model. Indeed, the AdG theory predicts that the brush 
height $L$ is given by $L \sim N \Gamma^{1 / 3}$ where $\mathrm{N}$ is the number of segments per chain and $\Gamma$ the grafting density.

Table 1: Brush layer thickness and grafting density obtained from AdG model (Eq. 1)

\begin{tabular}{rcccccc}
\hline & \multicolumn{3}{c}{ PBS } & \multicolumn{3}{c}{ Pure water } \\
\cline { 2 - 7 } & $\Gamma[\mathrm{nm}]^{-2}$ & $L[\mathrm{~nm}]$ & $\mathrm{R}^{2}$ & $\Gamma[\mathrm{nm}]^{-2}$ & $L[\mathrm{~nm}]$ & $\mathrm{R}^{2}$ \\
\hline $10 \mathrm{mg} / \mathrm{mL}$ & $1.5 \times 10^{-3}$ & 71 & 0.62 & $2.2 \times 10^{-3}$ & 77 & 0.51 \\
$100 \mathrm{mg} / \mathrm{mL}$ & $2.1 \times 10^{-3}$ & 70 & 0.95 & $3.4 \times 10^{-3}$ & 75 & 0.94 \\
\hline
\end{tabular}

Recent simulation studies have proposed that grafted BB polymers behave as elastic rods. ${ }^{49}$ AFM images confirmed that the persistence length of the polymer adsorbed on mica surfaces was similar to the contour length of the polymer (Figure 1B) therefore supporting this assumption. In this framework, the expected contributions to the total interaction energy between BB polymer coated surfaces are the bending energy of the rod ${ }^{49}$ and its conformational entropy. ${ }^{50}$ Considering these two contributions, the interaction forces can be written as:

$$
\frac{F_{N}}{R} \approx \mathrm{Ak}_{\mathrm{B}} T \frac{\Gamma^{2}}{D^{4}}+\mathrm{Bk}_{\mathrm{B}} T \Gamma \ln \left(\frac{2 L}{D}\right) \quad D<L \quad(\text { Eq. } 2)
$$

The first left-hand term in Eq. 2 represents the bending contribution of the BB polymer at the anchoring point (A being a prefactor depending on the brush architecture and $\Gamma$ the grafting density) ${ }^{49}$ and the second term, the entropic contribution of the non-adsorbed portion of the BB polymer ( $L$ being the height of the BB polymer layer and B being a prefactor close to unity). At low grafting density, Eq. 2 predicts that the entropic contribution is the main contribution to the long range forces. Therefore, under such conditions, the onset of the interaction forces is expected to depend weakly on the grafting density as observed experimentally (see Fig 3). In table 2 we show the fitted parameters using Eq. 2 (parameters for the entropic term only are shown for comparison purposes). As can be seen in figure $3 \mathrm{C}$ and $\mathrm{D}$ and Table 2, equation 2 
shows excellent agreement with the experimental data independantly of the ionic strength of the medium or the polymer concentration.

Table 2: Brush layer thickness and grafting density obtained from Eq. 2

\begin{tabular}{rccccccc}
\hline & \multicolumn{3}{c}{ PBS } & & \multicolumn{3}{c}{ Pure water } \\
\cline { 2 - 3 } \cline { 6 - 8 } & $\Gamma[\mathrm{nm}]^{-2}$ & $L[\mathrm{~nm}]$ & $\mathrm{R}^{2}$ & & $\Gamma[\mathrm{nm}]^{-2}$ & $L[\mathrm{~nm}]$ & $\mathrm{R}^{2}$ \\
\hline $10 \mathrm{mg} / \mathrm{mL}$ & $4.2 \times 10^{-2}$ & 65 & 0.95 & & $4.4 \times 10^{-2}$ & 60 & 0.91 \\
$100 \mathrm{mg} / \mathrm{mL}$ & $7.8 \times 10^{-2}$ & 67 & 0.97 & & $8.0 \times 10^{-2}$ & 67 & 0.97 \\
\hline
\end{tabular}

\section{Impact of aging on the BB polymer conformation at interfaces}

Using the framework of the rod-like behavior described by Eq. 2, we performed measurements of interactions forces between surfaces across aged BB polymer solutions. In this study, we used PBS as the solution medium and stored the polymer solutions at three different temperatures for a period of several weeks. Figure 4 presents the evolution of the measured interaction forces with the storage time at $4{ }^{\circ} \mathrm{C}, 22{ }^{\circ} \mathrm{C}$ and $37^{\circ} \mathrm{C}$. We can notice a systematic decrease of the onset of interaction, $D_{\text {onset }}=2 L$, with the storage time. The decrease of the onset of interaction increased significantly from low to high storage temperature. At high normal forces, the film thickness remained almost constant at $\sim 5 \mathrm{~nm}$ independently of the storage conditions. As shown in Fig. 4, the force profiles were adequately described by Eq. 2 allowing to determine accurately the BB polymer layer thickness, $L$, reported in Fig. 5. 

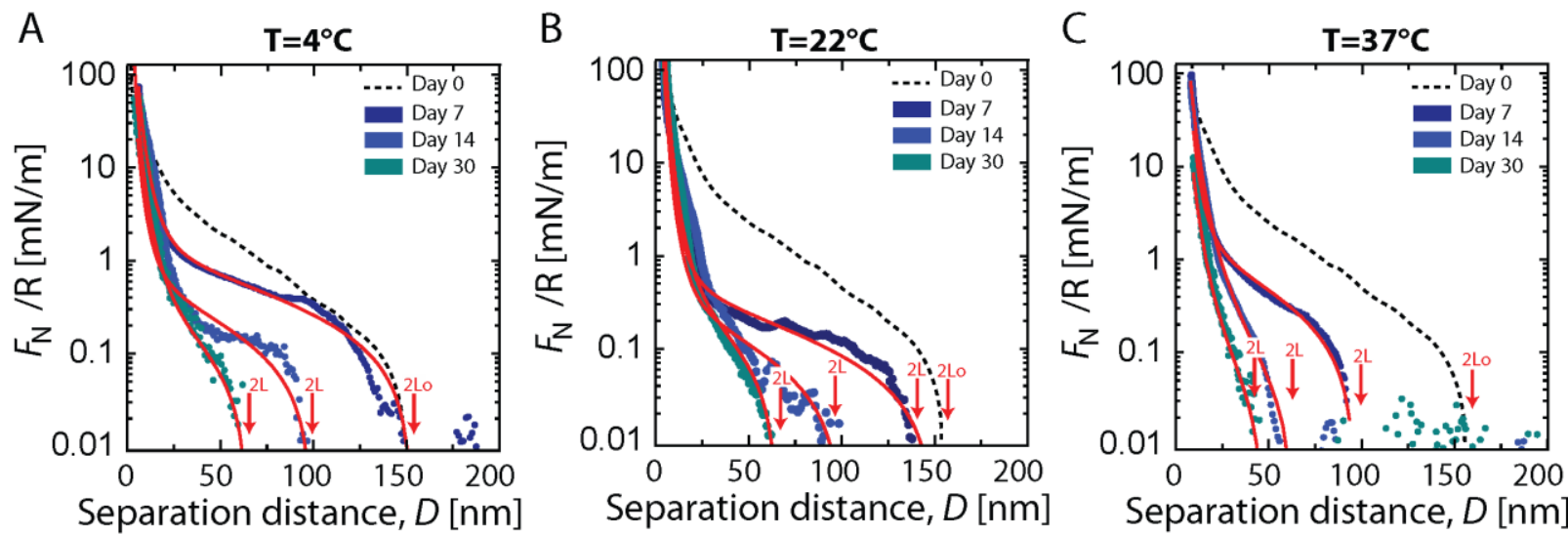

Figure 4. Interaction force profile between mica surfaces across a solution of $100 \mu \mathrm{g} / \mathrm{mL}$ BB polymer stored during 7,14 and 30 days in buffered saline at (A) $4^{\circ} \mathrm{C},(\mathrm{B}) 22^{\circ} \mathrm{C}$, and (C) $37^{\circ} \mathrm{C}$. Dashed line is day 0 $\mathrm{BB}$ interaction forces profiles extracted from figure 2 . Red lines are fittings obtained from equation 2.

The decay of the onset of interaction $L$ shown in Fig. 5 suggests a gradual change of the BB polymer size or a transition from a rigid to a more flexible BB polymer. Changes in the length of the BB polymer could be the result of a scission of the polymer backbone due to strong steric hindrance generated by the grafted side chains. On the other hand, a transition towards a more flexible BB polymer could be the product of the cleavage of lateral chains over time. Indeed, mean field theory ${ }^{1}$ predicts that the mean square size of a $\mathrm{BB}$ polymer chain, $\left\langle R^{2}\right\rangle$, which is, as a first order approximation, related to the onset of interaction $L\left(L^{2} \sim<R^{2}>\right)$ measured by SFA, is proportional to the side chain grafting density $z$, i.e. $\left\langle R^{2}\right\rangle \propto z^{\beta}$ with $\beta$ being close to unity. The decay of $L^{2}$ (normalized by the onset of interaction at $t=0, L_{0}$ ) was accurately fitted with a first order kinetic law at all temperatures except $37{ }^{\circ} \mathrm{C}$ At this temperature, a second distinct (much slower) kinetic process appears at incubation time longer than 15 days. From these data, the rate constant $k$ of the fast process only was estimated at the different temperatures tested and the corresponding half-life time of the fast process, $t_{1 / 2}$ was estimated to vary between 10 and 30 days depending on the storage temperature. 


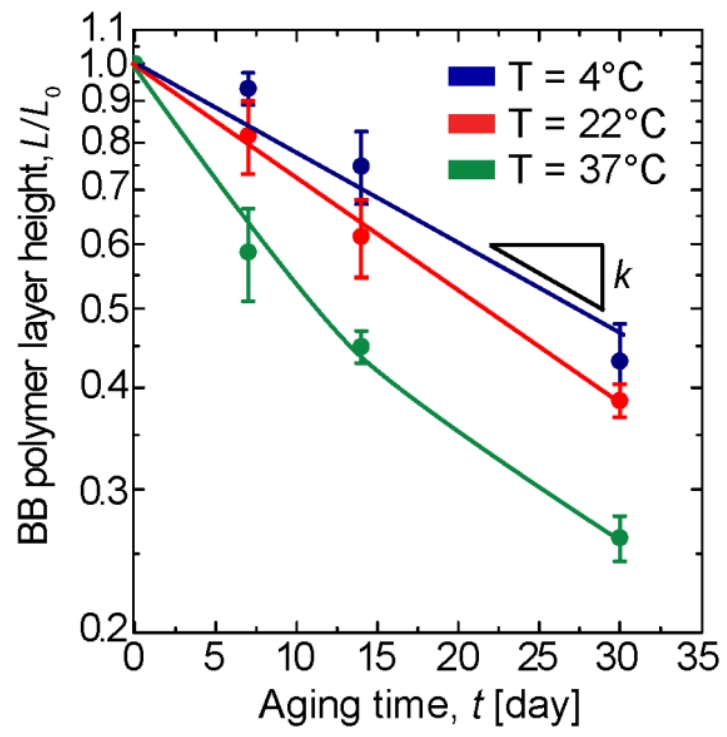

Figure 5. (A) Evolution of BB polymer length using SFA interaction onset and $B B$ fitting as a function of time at 4,22 and $37^{\circ} \mathrm{C}$. Solid lines are guide for the eyes.

In order to confirm the origin of the observed conformational changes of the BB polymers, we first measured the contour length of the BB polymer aged at $22{ }^{\circ} \mathrm{C}$ for 60 days by AFM imaging (Fig. 6A). No significant variation in the contour length, $L_{\mathfrak{c}}$, could be observed from the analysis of the AFM images (Fig. 6A and B). Some evidence of side-chains degrafting was obtained by aqueous GPC (in PBS) of the BB polymer solutions stored at different temperatures. The analysis revealed the appearance of a small population of low molecular weight chains at long storage time (peak 2 in Fig. 6C) alongside with a strong and constant peak corresponding to the BB polymer (peak 1). Peak 1 presents a small shoulder that appears at all incubation times and temperatures which was already present in the macroinitiator GPC trace (data not shown). The molecular weight of peak 2 obtained by GPC was close to the expected molecular mass of pMPC lateral chain, $M_{\mathrm{w}} \sim 20000 \mathrm{~g} / \mathrm{mol}$, and was independent of the storage temperature. This last observation confirmed that degrafting of the lateral chains indeed occurs and happens 
predominantly at the junction between the pendant chain and the BB polymer backbone. It could potentially be related to hydrolysis of the ester bonds linking side chains to the backbone. A similar observation was reported for brushes covalently linked to solid wafers in aqueous media. ${ }^{51}$ The areas of peak 2 allow to estimate a fraction of degrafted chains of less than $4 \%$ for all tested conditions after two months of storage. These results suggest that the half-life time of the BB polymer is at least 2 years. GPC results can be compared to previous SFA observations. For example, GPC data show a degrafting ratio of $3.5 \%$ after one month of aging at $4{ }^{\circ} \mathrm{C}$ which corresponds to a grafting ratio $z=0.55-0.04=0.51$. The expected value of the corresponding BB polymer layer $L / L_{0}$ is 0.96 which is significantly larger than the value of 0.43 obtained by SFA under the same aging conditions.

The differences between the SFA and GPC kinetic results could originate from small chains released from the BB polymer adsorbing preferentially on the surfaces therefore modifying the affinity / conformation of the BB polymer to the substrate. 


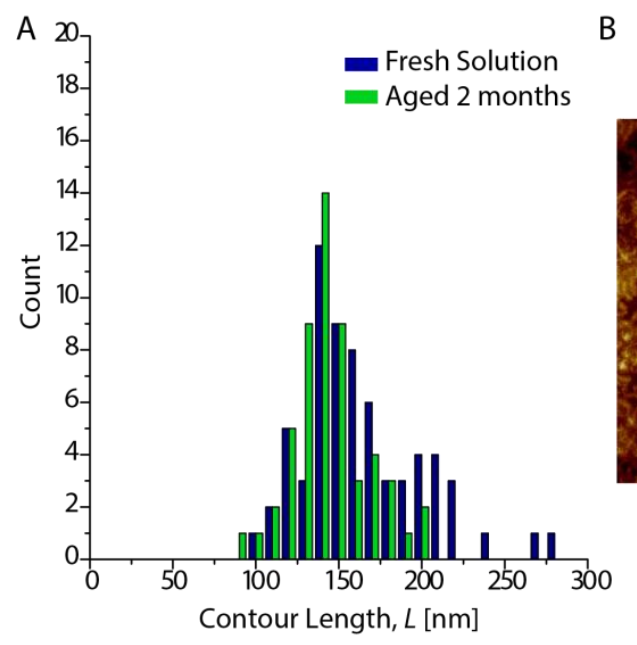

B
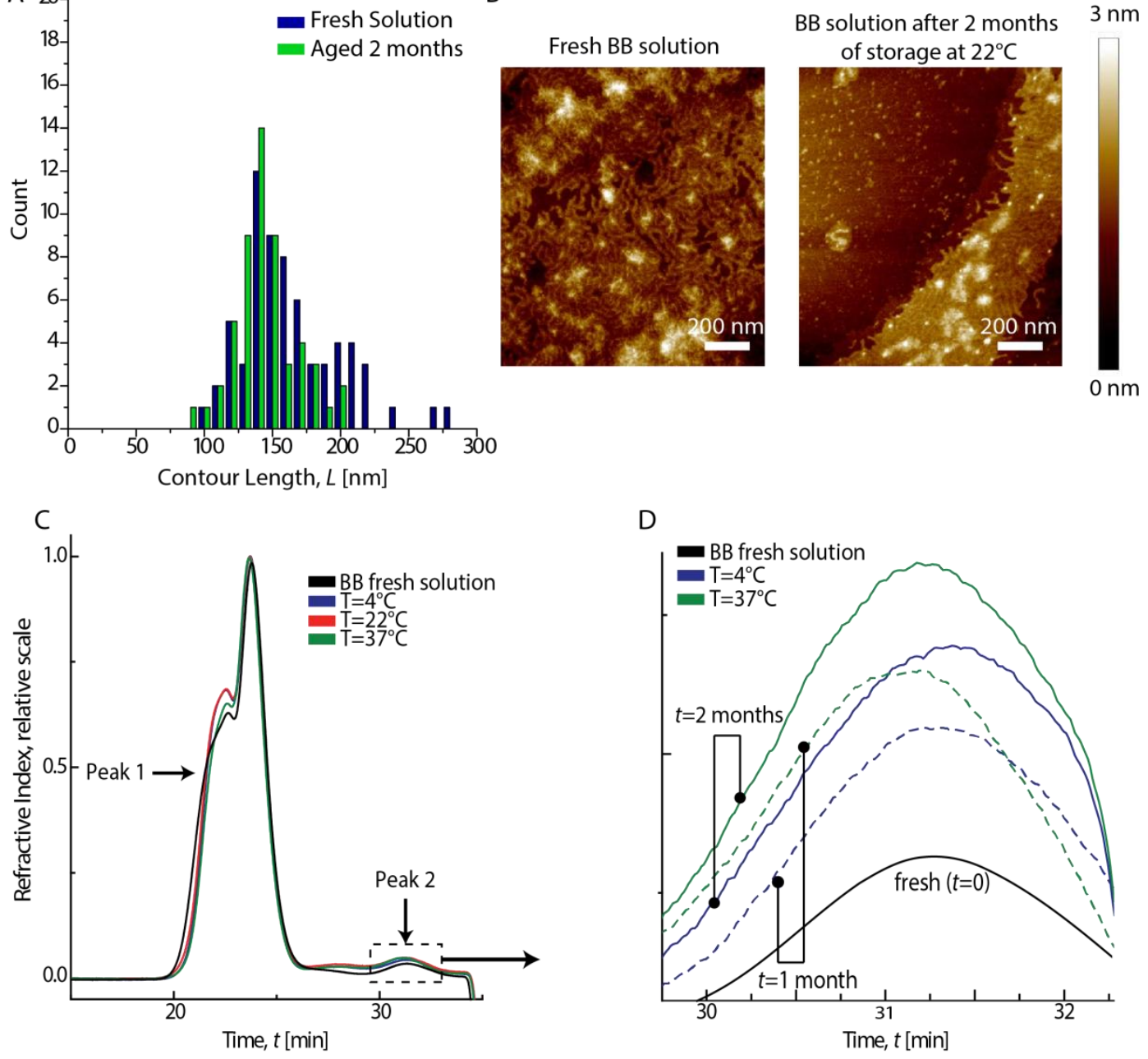

Figure 6. (A) BB polymer contour length distribution in PBS solution at $22^{\circ} \mathrm{C}$ measured immediately after dissolution and after 2 months of storage analyzed by AFM imaging, (B) Film of BB polymer chains dried on mica immediately after dissolution and after 2 months of storage. A $35 \mu \mathrm{g} / \mathrm{mL}$ BB polymer solution was used to generate the adsorbed films; (C) GPC traces of aged BB polymer in phosphate buffer, $\mathrm{pH}=$ 7.4 , at 4, 22 and $37^{\circ} \mathrm{C}$ compared to a fresh solution; (D) zoom-in of the elution chromatogram of peak 2 region showing the slight increase of the peak with storage time and temperature.

Lubrication and wear protection by aged BB polymers

Finally, we characterized the impact of the structural changes affecting the BB polymer on its frictional properties. Frictional properties of BB polymer fresh saline solution and stored 3 months at different temperatures were recorded using the SFA equipped with a bimorph slider designed for tribotesting. After storage, the solutions were placed in the SFA and friction forces, 
$F_{\mathrm{t}}$, were recorded at different applied normal forces $F_{\mathrm{N}}$ and constant sliding velocity. As shown in Fig. 7A, a linear relationship between the friction force and the normal force was observed for all samples tested. Such behavior was already reported in a previous report with a BB polymer with a triblock architecture designed to strongly adsorb on the substrate ${ }^{6}$. The linear relationship between $F_{\mathrm{t}}$ and $F_{\mathrm{N}}$, which is reminiscent of the peculiar rheological properties of the polymer under confinement, allows to extract the friction coefficient $(\mathrm{CoF})$ defined as $\mu=F_{\mathrm{t}} / F_{\mathrm{N}}$.

The measured $\mathrm{CoF}$ of the different solutions of $\mathrm{BB}$ polymer before and after the occurrence of damage were $\mu=0.031 \pm 0.002$ and $\mu=0.605 \pm 0.040$, respectively (Fig. 7B). Surprisingly, the $\mathrm{CoF}$ values before damage were independent of the storage temperature or storage time.

The wear initiation, characterized by the critical pressure of lubricating film rupture, $P^{*}$, was obtained through the analysis of the contact shape and separation between the surfaces assessed by multiple beam interferometry ${ }^{38}$. The measured values were $P^{*}=1.49 \pm 0.11 \mathrm{MPa}$ for BB fresh solution and $P^{*}=1.23 \pm 0.16$ for the $\mathrm{BB}$ solutions stored 3 months at different temperatures (Fig. 7C).
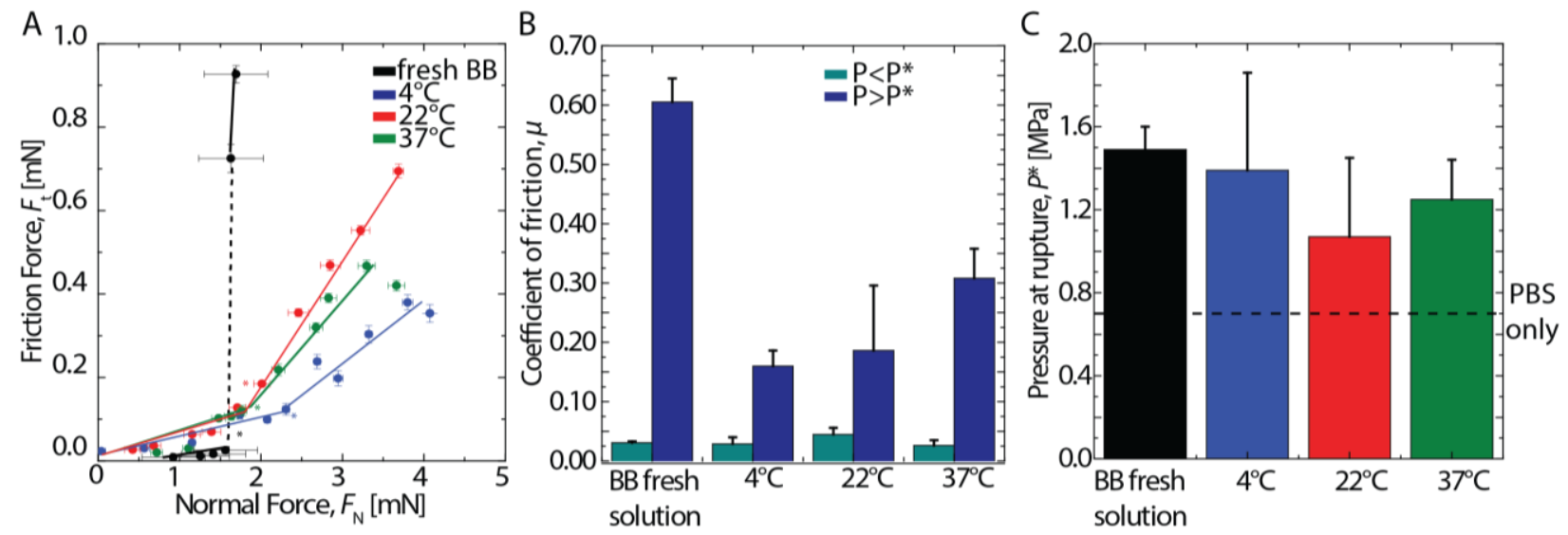

Figure 7. Tribology testing of $\mathrm{BB}$ polymer solutions after 3 months of storage in a phosphate buffer, $\mathrm{pH}$ 7.4 and $150 \mathrm{mM} \mathrm{NaCl}$, at 4,22 and $37^{\circ} \mathrm{C}$ are compared to a $\mathrm{BB}$ fresh solution: $(\mathrm{A})$ frictional forces, $F_{\mathrm{t}}$, as a function of normal force, $F_{N},(B)$ Friction coefficient, $\mu$, before $\left(P<P^{*}\right)$ and after $\left(P>P^{*}\right)$ wear initiation, (C) Evolution of the critical pressure at thin film rupture, $\mathrm{P}^{*}$, hallmark of wear initiation, with the 
different storage conditions.

This series of experiments demonstrated that the tribological properties of the BB polymer solution did not present any significant changes after 3 months of storage even if the polymer suffered detectable changes in its structure such as degrafting of lateral chains. We also noticed that the degrafting of the lateral chains of the BB polymer was associated to a less dramatic transition from smooth contact (no damage) to strongly damaged surfaces. A closer look of the evolution of the contact topography, assessed by the FECO shape, revealed that for the fresh BB solution, the wear initiation is not gradual but rather abrupt (Fig 8A a-c and B), due to the sudden formation of aggregates at the edge of the contact (highlighted by a white arrow in figure 8A-c). In contrast, the transition to wear was smoother for aged BB polymer solutions (Fig 8A d-f and B). A gradual accumulation of material inside the contact zone lead to an increase in separation distance before damage of mica occurred. Such smoother transition towards damage can be associated to the presence of degrafted pMPC chains covering the mica surface as previously suggested. The presence of these small and more mobile chains favors the accumulation of material in the contact instead of at the edge.
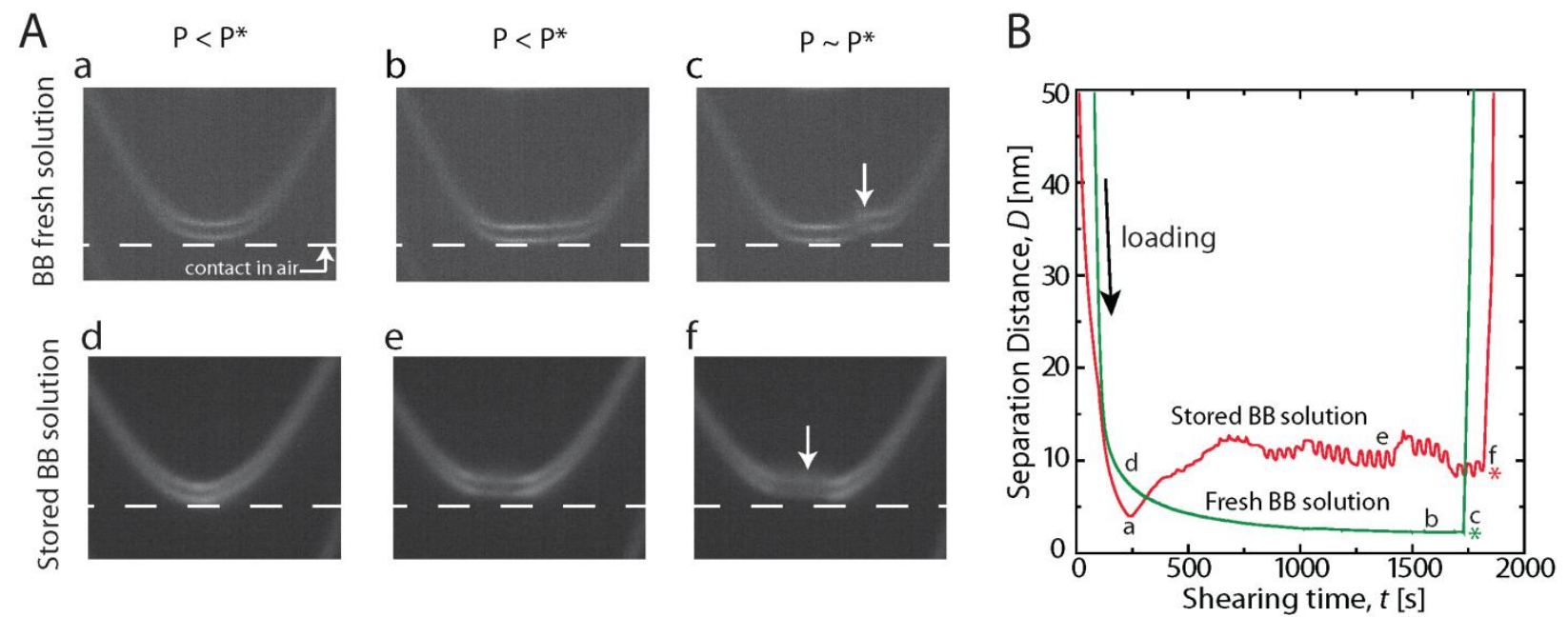

Figure 8. Evolution of FECO fringe shape with pressure for (A) BB polymer fresh solution (a-c), and 3 months aged at $22^{\circ} \mathrm{C} B B$ polymer solution ( $d-f$ ) White arrows, in $A-c$ and $f$, highlight the region of wear 
initiation; (B) Separation distance as a function of time for fresh and stored BB polymer solutions. Initiation of wear is indicated by an asterisk.

\section{Conclusions}

The present study has shown that BB polymers conformation at surfaces can evolve slowly with time and temperature. Careful experiments with the SFA allowed to quantify the dynamics of such gradual conformational changes and to correlate it with the adsorption of lateral chains on the substrate. These chains were slowly released from the BB polymer via hydrolysis of the linker functional group. The presence of these small chains on the surface did not have any impact on the tribological properties of the BB polymer. We observed no significant difference in the friction of coefficient or the critical pressure at onset of damage between all tested storage conditions. Altogether, the present study demonstrates that the BB polymer is stable enough in PBS to be considered as a potential injectable biolubricant for biomedical applications.

\section{Acknowledgements}

$\mathrm{XB}$ acknowledges the financial support from CIHR (CRC and Bridge grants) and NSERC (Discovery grant). JF is grateful to the Arthritis Society and the French Embassy (Frontenac scholarship) for financial support. BRS thanks the financial support of GRUM. KM acknowledges support from NSF (DMR 1501324 and DMR 1436219). We thank Agnes Crepet for her help with GPC experiments.

\section{Author contribution}

JF and BRS performed SFA experiments. GX synthesized the polymer. JF and BRS performed tribological work. JF carried out the stability experiments. JF, XB and KM wrote the manuscript. 
All the authors participated in the discussion of the data and in the production of the final version of the manuscript.

\section{Competing financial interests:}

The authors declare no competing financial interests. 
1. Paturej, J.; Sheiko, S. S.; Panyukov, S.; Rubinstein, M., Molecular structure of bottlebrush polymers in melts. Sci. Adv. 2016, 2, (11).

2. Denesyuk, N. A., Conformational properties of bottle-brush polymers. Phys. Rev. E: Stat. Nonlinear Soft Matter Phys. 2003, 67, (5 Pt 1), 051803.

3. Sheiko, S. S.; Sumerlin, B. S.; Matyjaszewski, K., Cylindrical molecular brushes: Synthesis, characterization, and properties. Prog. Polym. Sci. 2008, 33, (7), 759-785.

4. Zheng, X.; Zhang, C.; Bai, L.; Liu, S.; Tan, L.; Wang, Y., Antifouling property of monothiolterminated bottle-brush poly(methylacrylic acid)-graft-poly(2-methyl-2-oxazoline) copolymer on gold surfaces. J. Mater. Chem. B 2015, 3, (9), 1921-1930.

5. $\quad$ Daniel, W. F. M.; Burdynska, J.; Vatankhah-Varnoosfaderani, M.; Matyjaszewski, K.; Paturej, J.; Rubinstein, M.; Dobrynin, A. V.; Sheiko, S. S., Solvent-free, supersoft and superelastic bottlebrush melts and networks. Nat. Mater. 2016, 15, (2), 183-189.

6. Banquy, X.; Burdyńska, J.; Lee, D. W.; Matyjaszewski, K.; Israelachvili, J., Bioinspired Bottle-Brush Polymer Exhibits Low Friction and Amontons-like Behavior. J. Am. Chem. Soc. 2014, 136, (17), 61996202.

7. Li, X.; Prukop, S. L.; Biswal, S. L.; Verduzco, R., Surface Properties of Bottlebrush Polymer Thin Films. Macromolecules 2012, 45, (17), 7118-7127.

8. Sveinbjörnsson, B. R.; Weitekamp, R. A.; Miyake, G. M.; Xia, Y.; Atwater, H. A.; Grubbs, R. H., Rapid self-assembly of brush block copolymers to photonic crystals. Proc. Natl. Acad. Sci. U.S.A 2012, 109, (36), 14332-14336.

9. Sowers, M. A.; McCombs, J. R.; Wang, Y.; Paletta, J. T.; Morton, S. W.; Dreaden, E. C.; Boska, M. D.; Ottaviani, M. F.; Hammond, P. T.; Rajca, A.; Johnson, J. A., Redox-responsive branched-bottlebrush polymers for in vivo MRI and fluorescence imaging. Nat. Commun. 2014, 5, 5460.

10. Johnson, J. A.; Lu, Y. Y.; Burts, A. O.; Xia, Y.; Durrell, A. C.; Tirrell, D. A.; Grubbs, R. H., DrugLoaded, Bivalent-Bottle-Brush Polymers by Graft-through ROMP. Macromolecules 2010, 43, (24), 1032610335.

11. Venkataraman, S.; Hedrick, J. L.; Ong, Z. Y.; Yang, C.; Ee, P. L. R.; Hammond, P. T.; Yang, Y. Y., The effects of polymeric nanostructure shape on drug delivery. Adv. Drug Delivery Rev. 2011, 63, (14-15), 1228-1246.

12. Müllner, M.; Dodds, S. J.; Nguyen, T.-H.; Senyschyn, D.; Porter, C. J. H.; Boyd, B. J.; Caruso, F., Size and Rigidity of Cylindrical Polymer Brushes Dictate Long Circulating Properties In Vivo. ACS Nano 2015, 9, (2), 1294-1304.

13. Devarajan, P. V.; Jindal, A. B.; Patil, R. R.; Mulla, F.; Gaikwad, R. V.; Samad, A., Particle Shape: A New Design Parameter for Passive Targeting In Splenotropic Drug Delivery. J. Pharm. Sci. 2010, 99, (6), 2576-2581.

14. Zappone, B.; Ruths, M.; Greene, G. W.; Jay, G. D.; Israelachvili, J. N., Adsorption, lubrication, and wear of lubricin on model surfaces: polymer brush-like behavior of a glycoprotein. Biophys. J. 2007, 92, (5), 1693-708.

15. Jay, G. D.; Torres, J. R.; Warman, M. L.; Laderer, M. C.; Breuer, K. S., The role of lubricin in the mechanical behavior of synovial fluid. Proc. Natl. Acad. Sci. U.S.A 2007, 104, (15), 6194-9.

16. Chang, D. P.; Abu-Lail, N. I.; Guilak, F.; Jay, G. D.; Zauscher, S., Conformational Mechanics, Adsorption, and Normal Force Interactions of Lubricin and Hyaluronic Acid on Model Surfaces. Langmuir 2008, 24, (4), 1183-1193.

17. Coles, J. M.; Chang, D. P.; Zauscher, S., Molecular mechanisms of aqueous boundary lubrication by mucinous glycoproteins. Curr. Opin. Colloid Interface Sci. 2010, 15, (6), 406-416. 
18. Das, S.; Banquy, X.; Zappone, B.; Greene, G. W.; Jay, G. D.; Israelachvili, J. N., Synergistic interactions between grafted hyaluronic acid and lubricin provide enhanced wear protection and lubrication. Biomacromolecules 2013, 14, (5), 1669-77.

19. Majd, S. E.; Kuijer, R.; Kowitsch, A.; Groth, T.; Schmidt, T. A.; Sharma, P. K., Both Hyaluronan and Collagen Type II Keep Proteoglycan 4 (Lubricin) at the Cartilage Surface in a Condition That Provides Low Friction during Boundary Lubrication. Langmuir 2014, 14566-72.

20. Han, L.; Dean, D.; Ortiz, C.; Grodzinsky, A. J., Lateral Nanomechanics of Cartilage Aggrecan Macromolecules. Biophys. J. 2007, 92, (4), 1384-1398.

21. Seror, J.; Merkher, Y.; Kampf, N.; Collinson, L.; Day, A. J.; Maroudas, A.; Klein, J., Articular Cartilage Proteoglycans As Boundary Lubricants: Structure and Frictional Interaction of Surface-Attached Hyaluronan and Hyaluronan-Aggrecan Complexes. Biomacromolecules 2011, 12, (10), 3432-3443.

22. Roughley, P.; Mort, J., The role of aggrecan in normal and osteoarthritic cartilage. J. Exp. Orthop. 2014, 1, (1), 1-11.

23. Pettersson, T.; Naderi, A.; Makuska, R.; Claesson, P. M., Lubrication properties of bottle-brush polyelectrolytes: An AFM study on the effect of side chain and charge density. Langmuir 2008, 24, (7), 3336-3347.

24. Russano, D.; Carrillo, J. M. Y.; Dobrynin, A. V., Interaction between Brush Layers of Bottle-Brush Polyelectrolytes: Molecular Dynamics Simulations. Langmuir 2011, 27, (17), 11044-11051.

25. Liu, X.; Dedinaite, A.; Rutland, M.; Thormann, E.; Visnevskij, C.; Makuska, R.; Claesson, P. M., Electrostatically Anchored Branched Brush Layers. Langmuir 2012, 28, (44), 15537-15547.

26. Liu, X.; Thormann, E.; Dedinaite, A.; Rutland, M.; Visnevskij, C.; Makuska, R.; Claesson, P. M., Low friction and high load bearing capacity layers formed by cationic-block-non-ionic bottle-brush copolymers in aqueous media. Soft Matter 2013, 9, (22), 5361-5371.

27. Singh, M. K.; Ilg, P.; Espinosa-Marzal, R. M.; Kroeger, M.; Spencer, N. D., Polymer Brushes under Shear: Molecular Dynamics Simulations Compared to Experiments. Langmuir 2015, 31, (16), 4798-4805.

28. Milchev, A.; Paturej, J.; Rostiashvili, V. G.; Vilgis, T. A., Thermal Degradation of Adsorbed BottleBrush Macromolecules: A Molecular Dynamics Simulation. Macromolecules 2011, 44, (10), 3981-3987.

29. Sheiko, S. S.; Sun, F. C.; Randall, A.; Shirvanyants, D.; Rubinstein, M.; Lee, H.-i.; Matyjaszewski, K., Adsorption-induced scission of carbon-carbon bonds. Nature 2006, 440, (7081), 191-194.

30. Xia, Y.; Boydston, A. J.; Grubbs, R. H., Synthesis and Direct Imaging of Ultrahigh Molecular Weight Cyclic Brush Polymers. Angew. Chem. Int. Ed. 2011, 50, (26), 5882-5885.

31. Chen, K.; Susner, M. A.; Vyazovkin, S., Effect of the Brush Structure on the Degradation Mechanism of Polystyrene-Clay Nanocomposites. Macromol. Rapid Commun. 2005, 26, (9), 690-695.

32. $\mathrm{Xu}, \mathrm{L}$;; Crawford, K.; Gorman, C. B., Effects of Temperature and pH on the Degradation of Poly(lactic acid) Brushes. Macromolecules 2011, 44, (12), 4777-4782.

33. Lee, H. Y.; Han, L.; Roughley, P. J.; Grodzinsky, A. J.; Ortiz, C., Age-related nanostructural and nanomechanical changes of individual human cartilage aggrecan monomers and their glycosaminoglycan side chains. Journal of structural biology 2013, 181, (3), 264-73.

34. Faivre, J.; Shrestha, B. R.; Burdynska, J.; Xie, G.; Moldovan, F.; Delair, T.; Benayoun, S.; David, L.; Matyjaszewski, K.; Banquy, X., Wear Protection without Surface Modification Using a Synergistic Mixture of Molecular Brushes and Linear Polymers. ACS Nano 2017, 11, (2), 1762-69.

35. Wang, J.-S.; Matyjaszewski, K., Controlled/"living" radical polymerization. atom transfer radical polymerization in the presence of transition-metal complexes. J. Am. Chem. Soc. 1995, 117, (20), 56145615.

36. Matyjaszewski, K., Atom Transfer Radical Polymerization (ATRP): Current Status and Future Perspectives. Macromolecules 2012, 45, (10), 4015-4039. 
37. Nussbaum-Krammer, C. I.; Neto, M.; aacute; rio, F.; Brielmann, R.; e, M.; Pedersen, J. S.; Morimoto, R. I., Investigating the Spreading and Toxicity of Prion-like Proteins Using the Metazoan Model Organism C. elegans. J. Vis. Exp. 2015, (95).

38. Israelachvili, J. N., Thin film studies using multiple-beam interferometry. J. Colloid Interface Sci. 1973, 44, (2), 259-272.

39. Israelachvili, J.; Min, Y.; Akbulut, M.; Alig, A.; Carver, G.; Greene, W.; Kristiansen, K.; Meyer, E.; Pesika, N.; Rosenberg, K.; Zeng, H., Recent advances in the surface forces apparatus (SFA) technique. Rep. Prog. Phys. 2010, 73, (3).

40. Banquy, X.; Lee, D. W.; Das, S.; Hogan, J.; Israelachvili, J. N., Shear-Induced Aggregation of Mammalian Synovial Fluid Components under Boundary Lubrication Conditions. Adv. Funct. Mater. 2014, 24, (21), 3152-3161.

41. Lewis, A.; Tang, Y.; Brocchini, S.; Choi, J. W.; Godwin, A., Poly(2-methacryloyloxyethyl phosphorylcholine) for protein conjugation. Bioconjug. Chem. 2008, 19, (11), 2144-55.

42. Dedinaite, A., Biomimetic lubrication. Soft Matter 2012, 8, (2), 273-284.

43. Chen, M.; Briscoe, W. H.; Armes, S. P.; Klein, J., Lubrication at Physiological Pressures by Polyzwitterionic Brushes. Science 2009, 323, (5922), 1698-1701.

44. Kobayashi, M.; Terayama, Y.; Kikuchi, M.; Takahara, A., Chain dimensions and surface characterization of superhydrophilic polymer brushes with zwitterion side groups. Soft Matter 2013, 9, (21), 5138-5148.

45. Fleer, G.; Stuart, M. C.; Scheutjens, J.; Cosgrove, T.; Vincent, B., Polymers at interfaces. Springer Science \& Business Media: 1993.

46. Degennes, P. G., Polymers at an Interface - a Simplified View. Adv Colloid Interfac 1987, 27, (34), 189-209.

47. Tirrell, M.; Patel, S.; Hadziioannou, G., Polymeric amphiphiles at solid-fluid interfaces: Forces between layers of adsorbed block copolymers. Proc. Natl. Acad. Sci. U.S.A 1987, 84, (14), 4725-4728.

48. Marzolin, C.; Auroy, P.; Deruelle, M.; Folkers, J. P.; Léger, L.; Menelle, A., Neutron Reflectometry Study of the Segment-Density Profiles in End-Grafted and Irreversibly Adsorbed Layers of Polymer in Good Solvents. Macromolecules 2001, 34, (25), 8694-8700.

49. Russano, D.; Carrillo, J. M.; Dobrynin, A. V., Interaction between brush layers of bottle-brush polyelectrolytes: molecular dynamics simulations. Langmuir 2011, 27, (17), 11044-51.

50. Brangbour, C.; du Roure, O.; Helfer, E.; Demoulin, D.; Mazurier, A.; Fermigier, M.; Carlier, M. F.; Bibette, J.; Baudry, J., Force-velocity measurements of a few growing actin filaments. PLoS Biol 2011, 9, (4), e1000613.

51. Tugulu, S.; Klok, H.-A., Stability and nonfouling properties of poly (poly (ethylene glycol) methacrylate) brushes under cell culture conditions. Biomacromolecules 2008, 9, (3), 906-912. 

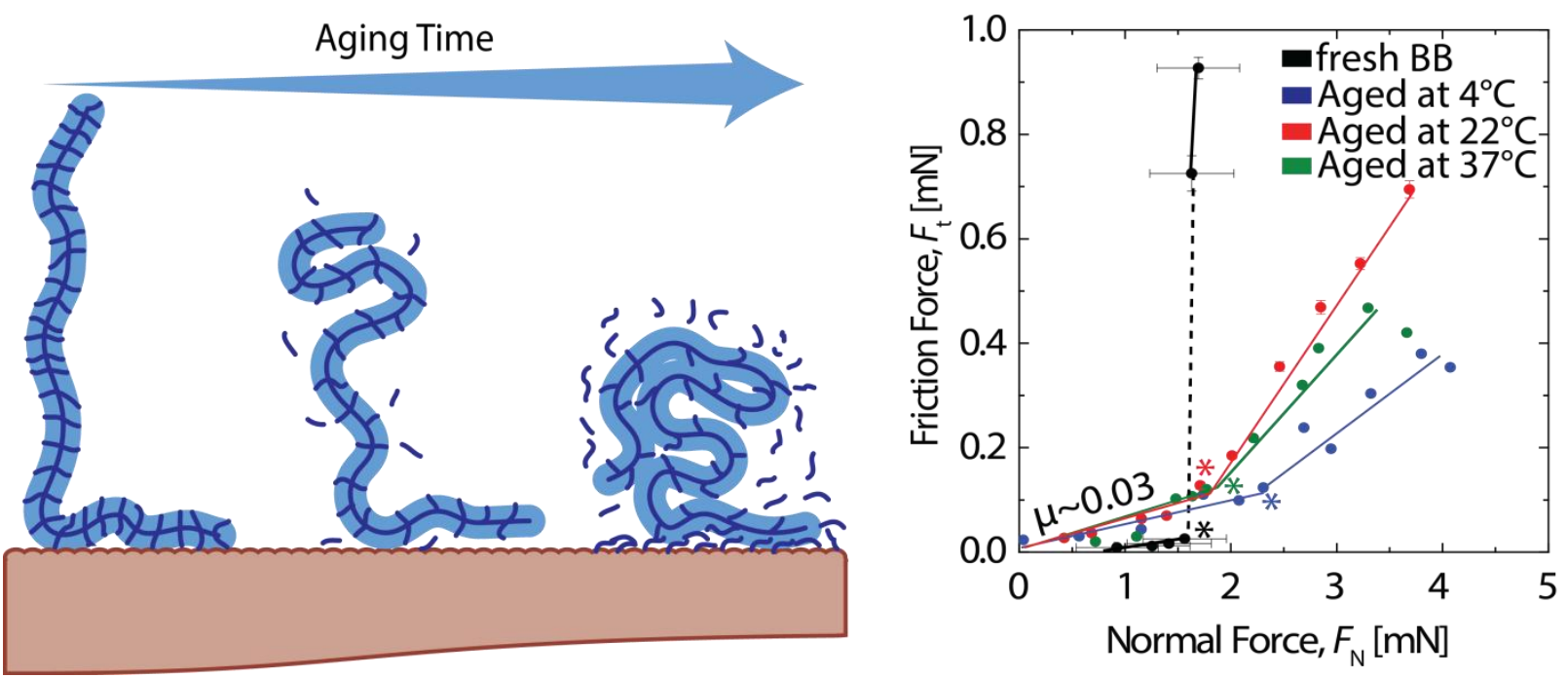